\title{
Review: there is no strong evidence to support antipsychotic combination therapy in schizophrenia
}

\author{
Freudenreich $O$, Goff DC. Antipsychotic combination therapy in schizophrenia. A review of efficacy and risks of current \\ combinations. Acta Psychiatr Scand 2002 Nov;106:323-30.
}

QUESTION: What are the benefits and risks of combining atypical antipsychotics with atypical or conventional antipsychcotics in schizophrenia?

\section{Design}

Systematic review with narrative synthesis.

\section{Data sources}

Studies were identified using Medline and hand searches of key articles, book chapters and bibliographies (search date not specified).

\section{Study selection}

Randomised trials, prospective observational studies and retrospective chart reviews published in English were eligible if they (1) compared combinations of atypical antipsychotic drugs with other atypical or conventional antipsychotics in schizophrenia; and (2) allowed an estimate of treatment response based on rating scales of psychopathology. 9 studies were identified with sample sizes ranging from 5 to 57.2 were randomised trials, 6 were prospective observational studies and 1 was a retrospective chart review.

\section{Data extraction}

Data were extracted on study type, antipsychotic combinations, dosage, outcome measures, number of participants and adverse effects. Main outcomes were response to treatment and adverse effects.

\section{Main results}

There is some empirical support for augmenting clozapine with more "tightly bound" D2 receptor antagonists such as risperidone. Studies were small and of limited rigour, however. The risks of augmentation have not been investigated systematically. There is little evidence about the benefits and harms of other combination regimens. The authors found no cost effectiveness analyses.

\section{Conclusions}

There is limited empirical evidence for the efficacy and safety of combining antipsychotics in schizophrenia.

\section{COMMENTARY}

Antipsychotics are commonly combined, although crosssectional surveys overestimate the prevalence because they include people receiving two antipsychotics while transferring from one to another. ${ }^{1}$ The risks of combining antipsychotics have recently been reviewed in detail, but evidence is still limited. ${ }^{2}$

The pharmacological rationale for combining antipsychotics is based on assumptions about complementary mechanisms of action. We may hypothesise that compounds that bind less tightly to D2 dopamine receptors (eg clozapine and quetiapine) become more effective when a strong $\mathrm{D} 2$ blocker is added. This could be tested using PET imaging of brain D2 dopamine receptor occupancy. An unanswered question is to what degree the use of such augmenting agents reintroduces the risk of EPS and tardive dyskinesia.

Given the variety of antipsychotics with a spectrum of adverse effect profiles, it may be possible to choose a partial replacement for a drug that is effective for a given individual, but which has tolerability or adverse effect problems at therapeutic doses. In general, however, clinicians prefer to substitute another antipsychotic with different adverse effects. Trying an alternate monotherapy is usually the wisest choice. For those who do not do well on alternate monotherapies, however, a combination which includes low doses of the efficacious but problematic antipsychotic may be reasonable. There are presently no studies to guide implementation of this approach, other than one where quetiapine partially substituted for clozapine. ${ }^{3}$

There is a tendency to believe that the widespread use of combination antipsychotics must mean that they work better than any monotherapy for some people. Aside from the case of augmentation of clozapine, however, this is arguable. As noted by Freudenreich $e t$ al, research in this area is sorely needed, but difficult to do well. While awaiting further evidence, clinicians need to consider the best interests of their patients. They can do so by first trying reasonable antipsychotic monotherapies, including clozapine, and by using combination antipsychotics only after finding that each component of the combination is inadequate without the other.

Alexander L Miller, MD University of Texas Health Science Center and San Antonio State Hospital San Antonio, Texas, USA

1 Covell NH, Jackson CT, Evans AC, Essock SM. Antipsychotic prescribing practices in Connecticut's public mental health Schizophrenia Bull 2002; 28: 17-29.

2 Miller AL, Craig CS. Combination antipsychotics: pros, cons, and questions. Schizophrenia Bull 2002; 28: 105-9.

Reinstein MJ, Sirotovskaya LA, Jones LE et al. Effect of clozapine-quetiapine combination therapy on weight and cozapine-quetiapine combination therapy on weight and glycaemic control:
Source of funding: None specified.

For correspondence: O Freudenreich, $M G H$ Schizophrenia Program, Freedom Trail Clinic, Boston. ofreud@massmed.org 